\title{
Courbes modulaires et 11-rang de corps quadratiques
}

\author{
Franck Leprévost
}

\section{TABLE DES MATIÈRES}

Introduction

1. Structure galoisienne de courbes modulaires

2. Le cas $p=23$

3. Méthode et Résultats

Remerciements

Bibliographie
Nous construisons 53 corps quadratiques imaginaires ayant un 11-rang égal à 3, et sept corps quadratiques réels de 11-rang égal à 2. Ce sont, à notre connaissance, les premiers exemples de tels corps.

We construct 53 imaginary quadratic fields of 11-rank equal to 3 , and seven quadratic fields of 11-rank equal to 2 . These appear to be the first examples of such fields.

\section{INTRODUCTION}

Soient $K$ un corps de nombres, $\mathrm{Cl}_{K}$ le groupe des classes d'idéaux de $K$, et $p$ un nombre premier. Le $p$-rang du groupe des classes d'idéaux de $K$ est, par définition, la dimension de $\mathrm{Cl}_{K} / p \mathrm{Cl}_{K}$ sur $\mathbf{F}_{p}$. Par abus de langage, nous entendrons par $p$-rang de $K$ le $p$-rang du groupe des classes d'idéaux de $K$.

Gauß a montré que le 2-rang d'un corps quadratique de discriminant $D$ est $t-1$ ou $t-2$, où $t$ est le nombre de facteurs premiers de $D$. Par conséquent, pour chaque entier $n \geq 1$, il existe une infinité de corps quadratiques réels (ou imaginaires) dont le 2-rang soit égal à $n$.

Shanks [1972] a trouvé les premiers exemples de corps quadratiques imaginaires dont le 3-rang est $\geq 3$. Depuis, divers auteurs (e.g. [Diaz y Diaz 1973 ; Craig 1977]) ont trouvé d'autres exemples de corps quadratiques de 3-rang supérieur à 3. Quer [1987] a trouvé trois corps quadratiques imaginaires dont le 3-rang est 6 . A chacun d'entre eux est associé, par un théorème de Scholz, un corps quadratique réel dont le 3 -rang est 5 .

Les cas $p=5$ et $p=7$ ont été étudiés par Mestre [1983; 1992]. Schoof [1983] a découvert le 
premier exemple de corps quadratique imaginaire dont le 5-rang est $\geq 4$, et Solderitsch [1977] le premier corps quadratique imaginaire dont le 7-rang est $\geq 3$. Pour d'autres exemples de tels corps, voir [Llorente et Quer].

Nous construisons ici 53 corps quadratiques imaginaires ayant un 11-rang égal à 3 , et sept corps quadratiques réels de 11-rang égal à 2. Ce sont, à notre connaissance, les premiers exemples de tels corps.

\section{STRUCTURE GALOISIENNE DE COURBES MODULAIRES}

Nous renvoyons à [Ling et Oesterlé 1991] pour cette partie. Soient $K$ un corps et $N$ un entier $\geq 1$. Pour $E$ une courbe elliptique définie sur $K$, notons $Y_{0}(N)(K)$ l'ensemble des classes de $\bar{K}$-isomorphisme de couples $(E, C)$, où $C$ est un sousgroupe de $E$ défini sur $K$ et cyclique d'ordre $N$; et notons $Y_{1}(N)(K)$ l'ensemble des classes de $\bar{K}$ isomorphisme de couples $(E, P)$, où $P$ est un point de $E$ défini sur $K$ et d'ordre $N$.

Soient

$$
\begin{aligned}
\Gamma & =\mathrm{SL}_{2}(\mathbf{Z}), \\
\Gamma_{0}(N) & =\left\{\left(\begin{array}{ll}
a & b \\
c & d
\end{array}\right) \in \Gamma ; c \equiv 0 \bmod N\right\}, \\
\Gamma_{1}(N) & =\left\{\left(\begin{array}{ll}
a & b \\
c & d
\end{array}\right) \in \Gamma_{0}(N) ; a \equiv d \equiv 1 \bmod N\right\} .
\end{aligned}
$$

Alors $Y_{0}(N)(\mathbf{C})$ et $Y_{1}(N)(\mathbf{C})$ sont isomorphes aux quotients de l'action des groupes $\Gamma_{0}(N) /\{ \pm 1\}$ et $\Gamma_{1}(N) /\{ \pm 1\}$, respectivement, sur le demi-plan de Poincaré. En rajoutant les pointes aux courbes $Y_{0}(N)(\mathbf{C})$ et $Y_{1}(N)(\mathbf{C})$ on obtient les compactifiées $X_{0}(N)(\mathbf{C})$ et $X_{1}(N)(\mathbf{C})$.

Enfin, notons $J_{1}$ et $J_{0}$ les jacobiennes des courbes $X_{1}(N)$ et $X_{0}(N)$.

\section{La courbe modulaire $\mathrm{X}_{0}(\mathrm{p})$}

Prenons $N=p$, où $p$ est un nombre premier impair, et posons $(p-1) / 12=a / b$, où $a$ et $b$ sont des entiers $\geq 1$ et premiers entre eux. Si

$$
\eta(z)=q^{1 / 24} \prod_{n \geq 1}\left(1-q^{n}\right)
$$

où $q=e^{2 i \pi z}$, la fonction

$$
f(z)=\left(\frac{\eta(z)}{\eta(p z)}\right)^{2 b}=q^{-a} \prod_{(n, p)=1}\left(1-q^{n}\right)^{2 b}
$$

est une fonction modulaire pour le groupe $\Gamma_{0}(p)$, de diviseur

$$
a[(0)-(\infty)],
$$

où $\infty$ et 0 représentent les pointes correspondantes de $X_{0}(p)$. On peut montrer que le sous-groupe engendré par le diviseur $(0)-(\infty)$ est exactement le groupe de torsion $C$ de la jacobienne de $X_{0}(p)(\mathbf{Q})$, et est d'ordre exactement $a$.

Supposons désormais $p \equiv-1 \bmod 12$ (ce que vérifie, par exemple, $p=23$ ), et considérons le revêtement

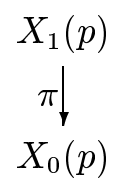

prolongeant celui défini en dehors des pointes par:

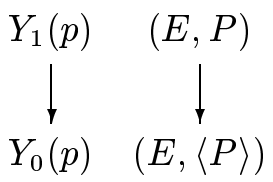

Ce revêtement est abélien cyclique de groupe de Galois $\mathbf{Z} / a \mathbf{Z}$, où $a=\frac{1}{2}(p-1)$.

Ce revêtement induit, par fonctorialité de $\mathrm{Pi}$ card :

$$
\pi^{*}: J_{0} \longrightarrow J_{1} .
$$

Le revêtement $\pi$ est cyclique de degré $a$. Donc [Ling et Oesterlé 1991, p. 172] il existe un sous-groupe $\Sigma$ (dit de Shimura) de la jacobienne de $X_{0}(p)\left(\mathbf{Q}\left(\mu_{a}\right)\right)$, isomorphe à $\mu_{a}$ en tant que $\operatorname{Gal}(\overline{\mathbf{Q}} / \mathbf{Q})$-module, et qui est le noyau de $\pi^{*}$. Ainsi obtient-on la suite exacte de $\operatorname{Gal}(\overline{\mathbf{Q}} / \mathbf{Q})$-modules :

$$
1 \rightarrow \mu_{a} \longrightarrow J_{0} \longrightarrow J_{1} .
$$

La jacobienne de la courbe modulaire $X_{0}(p)$ possède donc deux sous-groupes cycliques, rationnels sous l'action de $\operatorname{Gal}(\overline{\mathbf{Q}} / \mathbf{Q})$ et d'ordre $a$ : le groupe 
$C$ engendré par $(0)-(\infty)$ et le groupe $\Sigma$ de Shimura. $\operatorname{Gal}(\overline{\mathbf{Q}} / \mathbf{Q})$ agit trivialement sur $C$, comme $\mu_{a}$ sur $\Sigma$.

Supposons désormais a impair (donc $C \cap \Sigma=$ $\{0\}$ ) et premier. Les sous-groupes précédents permettent, dans certains cas, de construire, en utilisant la méthode décrite dans [Mestre 1982], des corps quadratiques dont le $a$-rang est $\geq 1$.

Soit

$$
1 \longrightarrow \operatorname{Ker}(\varphi) \longrightarrow C \stackrel{\varphi}{\longrightarrow} J_{0} \longrightarrow 1
$$

une suite exacte, où $C$ est une variété abélienne et $\varphi$ une isogénie définies sur $\mathbf{Q}$. Supposons $C$ semistable en tout $l$ premier. Soit $k$ un corps quadratique, $O_{k}$ son anneau des entiers, $C / o_{k}$ et $J_{0} / o_{k}$ les modèles de Néron de $C$ et $J_{0}$ sur $O_{k}$, et $\operatorname{Ker}(\varphi) / o_{k}$ la clôture schématique de $\operatorname{Ker}(\varphi) / k$ dans $C / o_{k}$. On a alors la suite exacte de schémas en groupes sur $O_{k}$

$$
1 \rightarrow \operatorname{Ker}(\varphi) / o_{k} \rightarrow C / o_{k} \rightarrow J_{0}^{\prime} / o_{k} \rightarrow 1,
$$

où $J_{0}^{\prime} / O_{k}$ est un sous-schéma en groupes ouvert de $J_{0} / O_{k}$, contenant la composante neutre de $J_{0} / O_{k}$ (ceci étend le lemme de [Mestre 1992, p. 371]).

Supposons dans la suite la courbe $X_{0}(p)$ hyperelliptique, d'équation

$$
y^{2}=f(x),
$$

où $f$ est un élément de $\mathbf{Q}[x]$ sans racines multiples. Notons $k$ le corps $\mathbf{Q}(\sqrt{f(x)})$ et $O_{k}$ l'anneau des entiers de $k$.

\section{Cas imaginaire}

Supposons que $k$ soit un corps quadratique imaginaire. Soit $B$ la variété abélienne quotient de $J_{0}$ par le sous-groupe d'ordre $a$ engendré par le diviseur $(0)-(\infty)$. On a une suite exacte:

$$
1 \rightarrow \mathbf{Z} / a \mathbf{Z} \rightarrow J_{0} \rightarrow B \longrightarrow 1
$$

qui, par dualité, donne une autre isogénie, définie sur $k$ :

$$
1 \rightarrow \mu_{a} \rightarrow{ }^{t} B \rightarrow J_{0} \rightarrow 1
$$

Posons $C={ }^{t} B$ et supposons $C$ semi-stable en tout nombre premier $l$. Notons $G$ le schéma en groupes quasi-fini clôture schématique de $\mu_{a / k}$ dans $C / O_{k}$. Alors on a, pour un certain sous-schéma en groupes $J_{0}^{\prime} / O_{k}$ ouvert de $J_{0} / o_{k}$ et contenant la composante neutre de $J_{0} / O_{k}$, la suite exacte de schémas en groupes sur $O_{k}$ :

$$
1 \longrightarrow G \longrightarrow C / o_{k} \longrightarrow J_{0}^{\prime} / o_{k} \longrightarrow 1 .
$$

En regardant cette suite exacte comme une suite exacte de faisceaux $\operatorname{sur} \operatorname{Spec}\left(O_{k}\right)$ pour la topologie fppf, on en déduit une injection:

$$
1 \longrightarrow J_{0}^{\prime}\left(O_{k}\right) / C\left(O_{k}\right) \longrightarrow \mathrm{H}^{1}\left(\operatorname{Spec}\left(O_{k}\right), G\right) .
$$

D'autre part, on a la suite exacte

$$
1 \longrightarrow G \longrightarrow \mu_{a} \rightarrow \mu_{a} . \longrightarrow 1
$$

où $\mu_{a}$. désigne un faisceau en gratte-ciel trivial en dehors des places de $k$ divisant $a$. D'où l'injection

$$
1 \rightarrow \mathrm{H}^{1}\left(\operatorname{Spec}\left(O_{k}\right), G\right) \longrightarrow \mathrm{H}^{1}\left(\operatorname{Spec}\left(O_{k}\right), \mu_{a}\right) .
$$

Enfin, $k$ étant un corps quadratique imaginaire, $\mathrm{H}^{1}\left(\operatorname{Spec}\left(O_{k}\right), \mu_{a}\right)$ s'injecte dans ${ }_{a} \mathrm{Cl}_{k}$, d'où l'existence d'un morphisme injectif

$$
J_{0}^{\prime}\left(O_{k}\right) / C\left(O_{k}\right) \longrightarrow{ }_{a} \mathrm{Cl}_{k}
$$

\section{Cas réel}

Supposons ici $k$ quadratique réel. La suite exacte

$$
1 \rightarrow \mu_{a} \longrightarrow J_{0} \stackrel{\pi^{*}}{\longrightarrow} J_{1}
$$

montre qu'il existe une variété abélienne $B=\operatorname{Im} \pi^{*}$ telle que l'on ait la suite exacte:

$$
1 \rightarrow \mu_{a} \longrightarrow J_{0} \longrightarrow B \longrightarrow 1,
$$

qui donne par dualité, en notant $C={ }^{t} B$ et $\varphi=$ ${ }^{t} \pi^{*}$ :

$$
1 \rightarrow \mathbf{Z} / a \mathbf{Z} \rightarrow C \stackrel{\varphi}{\longrightarrow} J_{0} \longrightarrow 1,
$$

(la variété abélienne $J_{0}$ étant une jacobienne, elle est isomorphe sur $\mathbf{Q}$ à sa duale).

Supposons $C$ semi-stable en tout nombre premier $l$. Pour un certain sous-schéma en groupes 
$J_{0}^{\prime} / o_{k}$ ouvert de $J_{0} / o_{k}$ et contenant la composante neutre de $J_{0} / o_{k}$, on a la suite exacte de schémas en groupes sur $O_{k}$ :

$$
1 \rightarrow \mathbf{Z} / a \mathbf{Z} \rightarrow C / o_{k} \rightarrow J_{0}^{\prime} / o_{k} \rightarrow 1,
$$

qui induit un homomorphisme de groupes:

$$
0 \longrightarrow J_{0}^{\prime}\left(O_{k}\right) / C\left(O_{k}\right) \longrightarrow \operatorname{Hom}\left(\mathrm{Cl}_{k}, \mathbf{Z} / a \mathbf{Z}\right),
$$

car

$$
\begin{aligned}
\mathrm{H}_{\mathrm{fppf}}^{1}\left(\operatorname{Spec}\left(O_{k}\right), \mathbf{Z} / a \mathbf{Z}\right) & =\mathrm{H}_{\mathrm{et}}^{1}\left(\operatorname{Spec}\left(O_{k}\right), \mathbf{Z} / a \mathbf{Z}\right) \\
& =\operatorname{Hom}\left(\mathrm{Cl}_{k}, \mathbf{Z} / a \mathbf{Z}\right) .
\end{aligned}
$$

Par suite, l'image réciproque par $\varphi$ de tout point de $J_{0}^{\prime}\left(O_{k}\right)$ engendre une extension de $k$, abélienne non ramifiée et de degré divisant $a$.

De manière concrète: soit $x$ un rationnel et $Q$ l'un des deux points de $X_{0}(p)$ d'abscisse $x$. Supposons que $Q$ ne se réduise pas modulo $p$ en le point singulier de $X_{0}(p) / \mathbf{F}_{p}$. Alors le théorème de Chevalley et Weil [1932] permet de montrer que l'extension $k\left(\varphi^{-1}(Q)\right) / k$, où $k=\mathbf{Q}(\sqrt{f(x)})$, est non ramifiée et de degré $a$.

\section{La courbe modulaire $X_{0}(2 p)$}

Si $N=N_{1} N_{2}$, où $N_{1}$ et $N_{2}$ sont des entiers $\geq 1$ et premiers entre eux, nous pouvons définir sur $\mathbf{Q}$ une involution $\omega_{N_{1}}^{N}$ (dite d'Atkin-Lehner) de $X_{0}(N)$, en posant, pour tout $(E, C) \in Y_{0}(N)$ :

$$
\omega_{N_{1}}^{N}((E, C))=\left(E / C_{N_{1}}, E_{N_{1}}+C_{N_{2}} / C_{N_{1}}\right),
$$

où $C_{N_{i}}$, pour $i=1,2$, est l'unique sous-groupe cyclique de $C$ d'ordre $N_{i}$, et $E_{N_{1}}$ est le groupe des points d'ordre $N_{1}$ de $E$.

La courbe modulaire $X_{0}(N)$ est donc revêtement de deux manières différentes de la courbe modulaire $X_{0}\left(N_{2}\right)$. En effet, un premier revêtement naturel, noté ici $\pi_{1}$, est donné, en dehors des pointes, par:

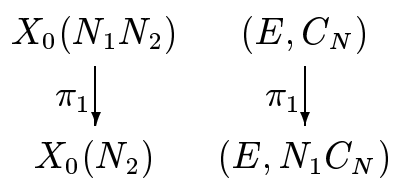

Un second revêtement, $\pi_{2}$, s'obtient en composant le revêtement ci-dessus avec l'involution $\omega_{N_{1}}^{N}$ d'Atkin-Lehner :

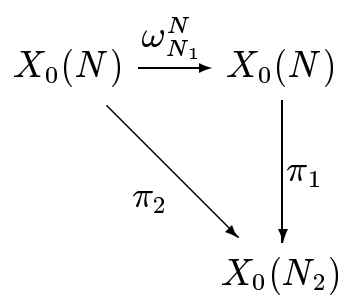

Si $N=2 p$, la courbe modulaire $X_{0}(2 p)$ est donc revêtement de deux manières de $X_{0}(p)$. De manière explicite, le morphisme $\pi_{1}$ est donné par

$$
\left(E, C_{2} C_{p}\right) \longrightarrow\left(E, C_{p}\right),
$$

et le morphisme $\pi_{2}$ par

$$
\left(E, C_{2} C_{p}\right) \rightarrow\left(E / C_{2}, C_{2} C_{p} / C_{2}\right) .
$$

Enfin l'involution $\omega_{2}^{2 p}$ de $X_{0}(2 p)$ est donnée par

$$
\left(E, C_{2} C_{p}\right) \longrightarrow\left(E / C_{2},\left[E[2] / C_{2}\right] \cdot C_{p}\right),
$$

où $E[2]$ désigne le noyau de la multiplication par 2 dans $E$.

Les revêtements $\pi_{1}$ et $\pi_{2}$ de $X_{0}(2 p)$ sur $X_{0}(p)$ sont indépendants. Le calcul montre en effet que les images réciproques par $\pi_{1}$ et $\pi_{2}$ de l'espace vectoriel $\Omega_{p}$ sont supplémentaires dans $\Omega_{2 p}$, où, pour $N=p$ ou $N=2 p, \Omega_{N}$ désigne l'espace des formes différentielles de première espèce de $X_{0}(N)$.

Soit $\left(E, C_{2} C_{p}\right)$ un élément de $X_{0}(2 p)(k)$ et $(E, P)$ un élément de $X_{1}(p)$ au dessus de $\pi_{1}\left(\left(E, C_{2} C_{p}\right)\right)$. En considérant l'isogénie de degré 2 définie sur $k$ :

$$
\psi: E \longrightarrow E / C_{2},
$$

on constate que $\psi(P)$ est un point de $E / C_{2}$, défini sur $k$ et qui engendre le sous-groupe $C_{2} C_{p} / C_{2}$. Par suite la jacobienne de $X_{0}(2 p)$ ne contient qu'une copie de $\Sigma$.

Une démonstration de cela se trouve également dans [Ling et Oesterlé 1991], que nous reproduisons ci-dessous. 
Nous avons vu que le revêtement

$$
\begin{gathered}
X_{1}(p) \\
\pi(p) \downarrow \\
X_{0}(p)
\end{gathered}
$$

induit, par fonctorialité de Picard, une suite exacte

$$
1 \longrightarrow \Sigma(p) \longrightarrow J_{0}(p) \stackrel{\pi^{*}(p)}{\longrightarrow} J_{1}(p) .
$$

De même, le revêtement

$$
\begin{gathered}
X_{0}(2 p) \\
\pi_{1} \downarrow \\
X_{0}(p)
\end{gathered}
$$

induit un morphisme

$$
J_{0}(p) \stackrel{\pi_{1}^{*}}{\longrightarrow} J_{0}(2 p) .
$$

Or, on a $\pi_{1}^{*}(\Sigma(p)) \subset \Sigma(2 p)$ [Ling et Oesterlé 1991, Th. 4, p. 175]. Mais

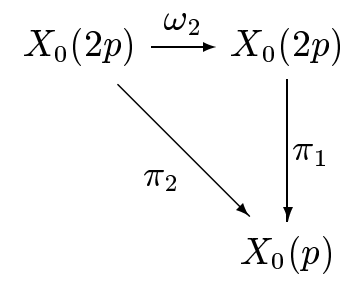

induit

$$
J_{0}(p) \stackrel{\pi_{2}^{*}}{\longrightarrow} J_{0}(2 p)
$$

et $\pi_{2}=\pi_{1} \circ \omega_{2}$ implique $\pi_{2}^{*}=\omega_{2}^{*} \circ \pi_{1}^{*}$, donc

$$
\pi_{2}^{*}(\Sigma(p))=\omega_{2}^{*}\left(\pi_{1}^{*}(\Sigma(p))\right) .
$$

Par suite,

$$
\pi_{2}^{*}(\Sigma(p)) \subset \omega_{2}^{*}(\Sigma(2 p))=\Sigma(2 p),
$$

car, d'après [Ling et Oesterlé 1991, Th. 3, p. 174], le sous-groupe de Shimura $\Sigma(2 p)$ est stable sous $\omega_{2}^{*}$.

Donc les images par $\pi_{1}^{*}$ et par $\pi_{2}^{*}$ du sous-groupe de Shimura de $J_{0}(p), \Sigma(p)$, sont des sous-groupes de $\Sigma(2 p)$, le sous-groupe de Shimura de $J_{0}(2 p)$. Enfin, [Ling et Oesterlé 1991, Cor. 1, p. 173] permet de montrer que l'ordre de $\Sigma(2 p)$ est encore $a$, ce qui établit que $J_{0}(2 p)$ ne contient qu'une copie de $\Sigma(p)$.
Par contre, on peut verifier que les pointes de $X_{0}(2 p)$ engendrent un sous-groupe de sa jacobienne qui, en tant que module galoisien, est isomorphe à $(\mathbf{Z} / a \mathbf{Z})^{2}$.

Si la courbe $X_{0}(2 p)$ est hyperelliptique, d'équation $y^{2}=g(x)$, considérons le point $Q$ de $X_{0}(2 p)$ d'abscisse un rationnel $x$. Supposons que $Q$ ne se réduise pas modulo $p$ en un point singulier de la courbe $X_{0}(2 p) / \mathbf{F}_{p}$. Soit $k=\mathbf{Q}(\sqrt{g(x)})$; si $k$ est un corps quadratique imaginaire, le $a$-rang de $k$ est supérieur ou égal à 2 . Si $k$ est un corps quadratique réel, on peut seulement affirmer que le $a$-rang de $k$ est supérieur ou égal à 1.

\section{LE CAS $p=23$}

\section{La courbe modulaire $X_{0}(23)(\mathbf{Q})$}

Considérons la courbe modulaire de genre deux, donc hyperelliptique, $X_{0}(23)(\mathbf{Q})$. Une équation de cette courbe est, par exemple,

$Y^{2}=X^{6}-14 X^{5}+57 X^{4}-106 X^{3}+90 X^{2}-16 X-19$

[Fricke 1928]. Cette courbe a bonne réduction partout sauf en 23 où la réduction du modèle ci-dessus est une union de deux droites:

$$
Y^{2} \equiv(X+2)^{2}(X+5)^{2}(X+9)^{2} \bmod 23,
$$

donc n'est pas irréductible. Il convient donc d'éclater les singularités.

Dans un premier temps, considérons le point singulier modulo 23 d'abscisse $X=-2$. Posons $X=$ $-2+23 t$. Ainsi obtient-on la courbe:

$$
\begin{aligned}
Y^{2}=23^{2}\left(529 t^{3}-207 t^{2}\right. & +26 t-1) \\
& \times\left(529 t^{3}-391 t^{2}+78 t-5\right) .
\end{aligned}
$$

Le changement $Y=23 y$ mène à l'équation $y^{2}=$ $f_{-2}(t)$, où

$$
\begin{aligned}
f_{-2}(t)=\left(529 t^{3}-207 t^{2}\right. & +26 t-1) \\
& \times\left(529 t^{3}-391 t^{2}+78 t-5\right) .
\end{aligned}
$$

Alors $y^{2} \equiv 4(t+2)(t+15) \bmod 23$; on a donc éclaté le point singulier modulo 23 d'abscisse $X=-2$. 
Plaçons-nous maintenant sur le point singulier modulo 23 d'abscisse $X=-5$. Posons pour ce faire $X=-5+23 u$. On obtient ainsi

$$
\begin{aligned}
Y^{2}=23^{2}\left(279841 u^{6}-535348 u^{5}+413678 u^{4}\right. \\
\left.-166658 u^{3}+37105 u^{2}-4342 u+209\right) .
\end{aligned}
$$

Si l'on pose $Y=23 y$, on se ramène à l'équation

$$
\begin{aligned}
y^{2}=279841 u^{6}-535348 u^{5}+413678 u^{4} \\
-166658 u^{3}+37105 u^{2}-4342 u+209,
\end{aligned}
$$

pour laquelle $y^{2} \equiv 6(u+10)^{2} \bmod 23$. Il faut faire un second éclatement en posant $u=-10+23 t$. On obtient $y^{2}=f_{-5}(t)$, avec $f_{-5}(t)$ égal à $23\left(148035889 t^{3}-198127428 t^{2}+88389023 t-13144019\right)$

$$
\times\left(12167 t^{3}-16468 t^{2}+7429 t-1117\right) .
$$

Formellement,

$$
\frac{f_{-5}(t)}{23} \equiv 3 \bmod 23
$$

le deuxième point singulier est bien éclaté.

Ôtons enfin la singularité modulo 23 d'abscisse $X=-9$ : en posant $X=-9+23 t$, nous obtenons $y^{2}=f_{-9}(t)$, avec

$$
\begin{aligned}
f_{-9}(t)=23\left(12167 t^{3}-20102 t^{2}+10649 t-1837\right) \\
\left(529 t^{3}-690 t^{2}+229 t-43\right) .
\end{aligned}
$$

Formellement on a

$$
\frac{f_{-9}(t)}{23} \equiv 9 \bmod 23
$$

et donc le dernier point singulier modulo 23 est éclaté.

\section{La courbe modulaire $X_{0}(46)(\mathbf{Q})$}

La courbe modulaire hyperelliptique $X_{0}(46)$ est revêtement de $X_{0}(23)$ de deux manières différentes : voir (1.1) et (1.2). Une équation de cette courbe est

$$
\begin{aligned}
& Y^{2}=\left(X^{3}+X^{2}+2 X+1\right)\left(X^{3}+4 X^{2}+4 X+8\right) \\
& \times\left(X^{6}+5 X^{5}+14 X^{4}+25 X^{3}+28 X^{2}+20 X+8\right)
\end{aligned}
$$

[Gonzàlez Rovira 1991], qui se réduit modulo 23 en

$$
\begin{aligned}
Y^{2} \equiv(X & +3)^{2}(X+5)^{2}(X+15)^{2} \\
& \times(X+16)^{2}(X+17)^{2}(X+18)^{2} \bmod 23 .
\end{aligned}
$$

Comme précédemment, nous éclatons la courbe $X_{0}(46)$ en les 6 points singuliers modulo 23 d'abscisses respectives $-5,-3,5,6,7$ et 8 modulo 23 . Nous obtenons ainsi, pour $m \in\{-5,-3,5,6,7,8\}$, les équations $y^{2}=g_{m}(t)$, où les polynômes $g_{m}(t)$ sont donnés ci-dessous.

$$
\begin{aligned}
g_{-5}(t)=23( & \left.12167 t^{3}-7406 t^{2}+1541 t-109\right)\left(12167 t^{3}-5819 t^{2}+897 t-37\right) \\
& \times\left(6436343 t^{6}-6996025 t^{5}+3212088 t^{4}-796145 t^{3}+112194 t^{2}-8510 t+271\right) \\
g_{-3}(t)=23 & \left(529 t^{3}-184 t^{2}+23 t-1\right)\left(12167 t^{3}-2645 t^{2}+161 t+5\right) \\
& \times\left(148035889 t^{6}-83672459 t^{5}+20708234 t^{4}-2834911 t^{3}+224296 t^{2}-9614 t+173\right) \\
g_{5}(t)=\left(529 t^{3}+368 t^{2}+87 t+7\right)\left(529 t^{3}+437 t^{2}+119 t+11\right) & \\
\times & \left(148035889 t^{6}+225272005 t^{5}+143838274 t^{4}+49337185 t^{3}+9589712 t^{2}+1001650 t+43933\right) \\
g_{6}(t)=23 & \left(12167 t^{3}+10051 t^{2}+2806 t+265\right)\left(12167 t^{3}+11638 t^{2}+3680 t+392\right) \\
& \times\left(6436343 t^{6}+11473481 t^{5}+8565568 t^{4}+3428449 t^{3}+776066 t^{2}+94208 t+4792\right) \\
g_{7}(t)=23 & \left(12167 t^{3}+11638 t^{2}+3749 t+407\right)\left(529 t^{3}+575 t^{2}+207 t+25\right) \\
& \times\left(148035889 t^{6}+302508121 t^{5}+258573084 t^{4}+118348409 t^{3}+30594186 t^{2}+4235726 t+245393\right) \\
g_{8}(t)=23 & \left(12167 t^{3}+13225 t^{2}+4830 t+593\right)\left(12167 t^{3}+14812 t^{2}+5980 t+808\right) \\
& \times\left(6436343 t^{6}+14831573 t^{5}+14284058 t^{4}+7359977 t^{3}+2140012 t^{2}+332948 t+21656\right)
\end{aligned}
$$

Polynômes utilisés pour l'éclatement des singularités de la courbe $X_{0}(46)$. 


\section{MÉTHODE ET RÉSULTATS}

Soient $a_{k}$ et $b_{k}$ les racines réelles de $f_{k}$, où $k=$ $-9,-5,-2$, et $c_{m}$ et $d_{m}$ les racines réelles de $g_{m}$, où $m=-5,-3,5,6,7,8$. Substituons dans $f_{k}$ un nombre rationnel $t=i / j$, où $j$ est premier à 23 et $a_{k}<t<b_{k}$ : le 11-rang du corps quadratique imaginaire $\mathbf{Q}\left(\sqrt{f_{k}(t)}\right)$ est $\geq 1$. De même, par substitution dans $g_{m}$ de nombres rationnels convenables, tels que $c_{m}<t<d_{m}$, l'on obtient des corps quadratiques imaginaires $\mathbf{Q}\left(\sqrt{g_{m}(t)}\right)$ dont le 11-rang est $\geq 2$.

Il n'était pas désespéré de trouver, par une recherche systématique dans ces familles, des corps quadratiques imaginaires ayant un 11-rang $\geq 3$.

Dans la pratique, on a imposé aussi la condition $1 \leq j \leq B$, où $B$ est une borne convenablement choisie. Pour chaque valeur de $t$ ainsi obtenue, on a calculé de discriminant $d$ correspondant. Pour les discriminants appartenant à $\left[-10^{20},-2510^{6}\right]$, dans le cas des $f_{k}$, ou à $\left[-10^{23},-2510^{6}\right]$, dans le cas des $g_{k}$, on a alors calculé le 11-rang de $\mathbf{Q}(\sqrt{d})$. (La borne supérieure de ces intervalles est imposée par les travaux de Buell [1987].) L'implémentation de cette méthode sera considerée ci-dessous.

Le tableau 1 fournit le nombre de corps quadratiques imaginaires obtenus à l'aide de chaque polynôme $f_{k}$ ou $g_{k}$, et le nombre de ceux qui ont 11-rang égal à $1,2,3$.

Nous avons également cherché, à l'aide des trois courbes associées à $X_{0}(23)$, des exemples de corps quadratiques réels ayant un 11-rang $\geq 2$. Pour ce faire, nous avons substitué dans $f_{k}$ des rationnels $t=i / j$, où $-B \leq j \leq B, j$ non nul premier à 23 , $1 \leq i \leq B, i$ premier avec $j$ tel que $i<a_{k} j$ ou $i>$ $b_{k} j$, en ne retenant que les discriminants $\leq 10^{15}$. Le tableau 2 résume nos résultats dans cette direction (la même méthode, appliquée aux courbes issues de $X_{0}(46)$, a donné des discriminants dont la taille rendait le temps de calcul prohibitif par rapport au résultat escompté).

Le tableau 3 contient l'information suivante pour chaque corps imaginaire de 11-rang égal a 3 : le discriminant $d$; le polynôme et les valeurs de $i$ et

\begin{tabular}{|lrrrrr|}
\hline & \multicolumn{1}{c}{$B$} & \multicolumn{1}{c}{1} & \multicolumn{1}{c}{2} & \multicolumn{1}{c|}{3} & \multicolumn{1}{c|}{ total } \\
\hline$f_{-2}$ & 2000 & 325073 & 35545 & 29 & 360647 \\
$f_{-5}$ & 10000 & 48672 & 5390 & 7 & 54069 \\
$f_{-9}$ & 2000 & 115775 & 13065 & 9 & 128849 \\
\hline$g_{-5}$ & 5000 & & 85 & 2 & 87 \\
$g_{-3}$ & 5000 & & 163 & 2 & 165 \\
$g_{5}$ & 5000 & & 241 & 3 & 244 \\
$g_{6}$ & 5000 & & 159 & 1 & 160 \\
$g_{7}$ & 5000 & & 163 & 2 & 165 \\
$g_{8}$ & 5000 & & 159 & 1 & 160 \\
\hline
\end{tabular}

TABLEAU 1. Nombre de corps quadratiques imaginaires de la forme $\mathbf{Q}\left(\sqrt{f_{k}(t)}\right)$ ou $\mathbf{Q}\left(\sqrt{g_{k}(t)}\right)$, avec $t=i / j$ et $j \leq B$ premier à 23 . Le nombre de corps ayant 11-rang 1, 2, et 3 est également indiqué.

\begin{tabular}{|rrrrr|}
\hline & $B$ & 1 & 2 & total \\
\hline$f_{-2}$ & 20 & 446 & 4 & 450 \\
$f_{-5}$ & 50 & 70 & 0 & 70 \\
$f_{-9}$ & 20 & 288 & 3 & 291 \\
\hline
\end{tabular}

TABLEAU 2. Nombre de corps quadratiques réels obtenus à l'aide des polynômes $f_{k}$.

$j$ qui ont permis de trouver $d$, selon le procédé cidessus ; et la structure du groupe des classes. (Dans la dernière colonne, chaque expression entre parenthèses désigne un group cyclique, de sorte que $(2)^{4}\left(3^{2}\right)(11)^{3}$ doit être interpreté comme le groupe $(\mathbf{Z} / 2 \mathbf{Z})^{4} \times(\mathbf{Z} / 9 \mathbf{Z}) \times(\mathbf{Z} / 11 \mathbf{Z})^{3}$. $)$ Les discriminants obtenus avec $g_{7}(t)$ et $g_{8}(t)$, étant les mêmes que ceux obtenus, respectivement, avec $g_{-3}(t)$ et $g_{6}(t)$, ne sont donc pas reproduits.

Le tableau 4 comporte les mêmes données pour les corps quadratiques réels de de 11-rang égal a 2 , le groupe des classes étant pris au sens restreint.

\section{Implémentation}

Nous avons exploité pour nos calculs deux programmes écrits en langage $\mathrm{C}$ et utilisant la bibliothèque PARI [Batut et al. 1992].

Le premier construit les discriminants satisfaisant les conditions décrites ci-dessus, et les stocke dans des fichiers, un pour chaque $f_{k}$ ou $g_{k}$. 


\begin{tabular}{|c|c|c|c|c|}
\hline$d$ & $k$ & $i$ & $j$ & groupe de classes \\
\hline 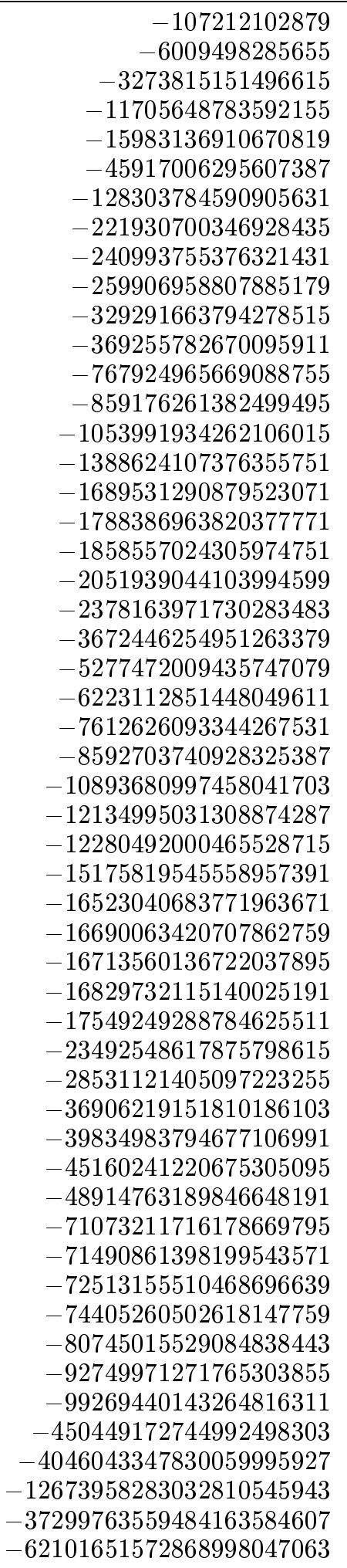 & $\begin{array}{l}f_{-2} \\
f_{-2} \\
f_{-2} \\
f_{-2} \\
f_{-2} \\
f_{-2} \\
f_{-9} \\
f_{-2} \\
f_{-2} \\
f_{-9} \\
f_{-9} \\
f_{-2} \\
f_{-2} \\
f_{-2} \\
f_{-2} \\
g_{-5} \\
f_{-5} \\
f_{-} \\
f_{-} \\
f_{-2} \\
f_{-2} \\
f_{-2} \\
f_{-2} \\
f_{-5} \\
f_{-} \\
g_{-} \\
g_{5} \\
g_{-}\end{array}$ & $\begin{array}{r}23 \\
35 \\
205 \\
106 \\
74 \\
138 \\
119 \\
266 \\
181 \\
783 \\
133 \\
189 \\
300 \\
215 \\
211 \\
3 \\
1065 \\
179 \\
169 \\
347 \\
274 \\
294 \\
337 \\
821 \\
532 \\
1283 \\
4029 \\
403 \\
338 \\
1501 \\
351 \\
361 \\
466 \\
1490 \\
361 \\
379 \\
679 \\
431 \\
89 \\
669 \\
458 \\
450 \\
606 \\
481 \\
713 \\
474 \\
-17 \\
-24 \\
\end{array}$ & $\begin{array}{r}152 \\
401 \\
1006 \\
293 \\
895 \\
523 \\
158 \\
1593 \\
464 \\
1429 \\
223 \\
1613 \\
1637 \\
622 \\
1873 \\
25 \\
2384 \\
303 \\
1926 \\
1864 \\
1099 \\
1175 \\
1508 \\
1827 \\
1781 \\
2877 \\
9082 \\
662 \\
1149 \\
3362 \\
1097 \\
955 \\
1229 \\
3339 \\
1259 \\
1322 \\
1502 \\
1716 \\
81 \\
1454 \\
1169 \\
1279 \\
1337 \\
1905 \\
1526 \\
1229 \\
1507 \\
60 \\
739 \\
\end{array}$ & 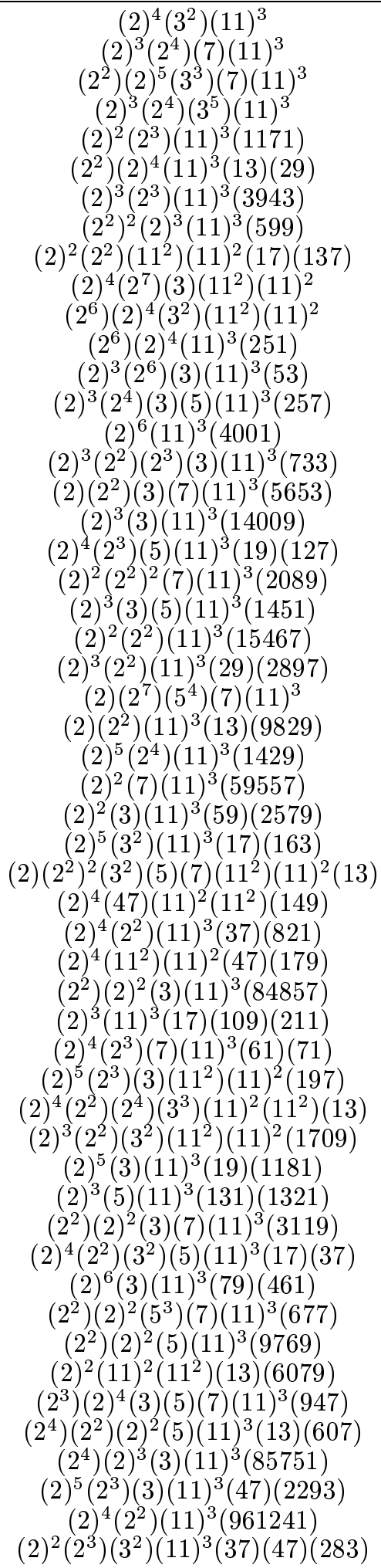 \\
\hline
\end{tabular}

TABLEAU 3. Données pour les 53 corps quadratiques imaginaires ayant 11-rang égal à 3 . 


\begin{tabular}{|r|c|r|r|c|}
\hline$d$ & $k$ & $i$ & $j$ & groupe de classes \\
\hline 317019341 & $f_{-2}$ & 2 & -7 & $(2)(11)^{2}$ \\
78990264001 & $f_{-2}$ & 7 & -6 & $(2)^{2}(11)^{2}$ \\
3628885436065 & $f_{-9}$ & 2 & -7 & $(2)^{3}(11)^{2}$ \\
11153056789873 & $f_{-2}$ & 17 & -8 & $(2)(3)(11)^{2}$ \\
27362217255701 & $f_{-2}$ & 18 & -19 & $(11)^{2}$ \\
39112346819681 & $f_{-9}$ & 9 & 2 & $(2)\left(2^{2}\right)(11)^{2}$ \\
720826508942753 & $f_{-9}$ & 15 & 4 & $(2)^{2}\left(2^{2}\right)(11)^{2}$ \\
\hline
\end{tabular}

TABLEAU 4. Données pour les sept corps quadratiques réels ayant 11-rang égal à 2 .

Le second est conçu sur le modèle «maître-esclave ». Le programme-maître lit dans les fichiers les discriminants et les distribue sur différentes machines. Le programme-esclave, exécuté sur ces machines, calcule, à la réception d'un discriminant $d$, le nombre de classes et le 11-rang du corps quadratique de discriminant $d$. Il retourne alors au programme-maître les quantités $d$ et le rang. Celuici range $d$ dans différents fichiers selon la valeur du rang.

Voici comme fonctionne le programme-esclave. Nous utilisons la procédure classno (basée sur l'algorithme de [Shanks 1971] et implémentée dans le système PARI) pour déterminer l'ordre du groupe des classes. Nous construisons ensuite un nombre, inférieur ou égal à 3 , de formes quadratiques indépendantes et d'ordre 11.

Enfin la structure du groupe des classes des corps quadratiques imaginaires de discriminants $d$ et de 11-rang égal à 3 a été obtenue, sous PARI-GP, avec la procédure buchimag (fondée, ainsi que la procédure buchreal, sur l'algorithme sous-exponentiel de Buchmann); nous avons examiné, pour chaque tel $d$, la concordance du nombre de classes obtenu via la procédure classno avec celui obtenu via la procédure buchimag.

Dans le cas des corps réels, nous n'avons pas eu recours au calcul distribué. Pour chacun des 811 discriminants retenus, nous avons déterminé la structure du groupe des classes correspondant (au sens restreint) grâce à la procédure buchreal.

Nous avons utilisé une implémentation distribuée sur vingt stations de travail Sparc de l'Ecole Normale Supérieure, en adaptant le programme conçu (et utilisé dans [Fermigier 1992]) par Stéfane Fermigier. Les calculs ont nécessité moins d'une semaine.

\section{REMERCIEMENTS}

Je tiens à remercier Stéfane Fermigier pour m'avoir permis d'utiliser son programme de calculs distribués (voir le paragraphe 3), et Jean-François Mestre, mon directeur de thèse, pour l'aide considérable et les encouragements qu'il m'a prodigués dans ce travail.

Les diagrammes de cet article ont été realisés à l'aide du programme diagrams.tex, conçu par Paul Taylor.

\section{BIBLIOGRAPHIE}

[Batut et al. 1992] C. Batut, D. Bernardi, H. Cohen and M. Olivier, User's Guide to PARI-GP, 1992. Ce manuel fait partie de la distribution du programme, disponible sur le serveur snekkar.ens.fr.

[Buell 1987] D. A. Buell, "Class groups of quadratic fields II", Math. Comp. 48 (1987), 85-93.

[Chevalley et Weil 1932] C. Chevalley et A. Weil, "Un théorème d'arithmétique sur les courbes algébriques", Comptes R. Acad. Sci. Paris 195 (1932), 570-572.

[Diaz y Diaz 1973] F. Diaz y Diaz, "On some families of imaginary quadratic fields", Math. Comp. 32 (1973), 636-650.

[Fermigier 1992] S. Fermigier, "Un exemple de courbe elliptique définie sur $\mathbf{Q}$ de rang $\geq 19$ ", Comptes $R$. Acad. Sci. Paris 315 (1992), 719-722.

[Fricke 1928] R. Fricke, Lehrbuch der Algebra, 3.ter Band, Vieweg, Braunschweig, 1928.

[Gonzàlez Rovira 1991] J. Gonzàlez Rovira, "Equations of hyperelliptic modular curves", Ann. Inst. Fourier, 41(4) (1991), 779-795.

[Ling et Oesterlé 1991] S. Ling et J. Oesterlé, "The Shimura subgroup of $J_{0}(N)$ ", pp. 171-203 in Courbes modulaires et courbes de Shimura, Astérisque 196197 (1991).

[Llorente et Quer] P. Llorente et J. Quer, Tables (non publié). 
[Mestre 1982] J.-F. Mestre, "Groupes de classes d'idéaux non cycliques de corps de nombres", Séminaire de Théorie des Nombres de Paris (1982), 189200.

[Mestre 1983] J.-F. Mestre, "Courbes elliptiques et groupes de classes de certains corps quadratiques", J. reine angew. Math. 343 (1983), 23-35.

[Mestre 1992] J.-F. Mestre, "Corps quadratiques dont le 5-rang du groupe des classes est $\geq 3$ ", Comptes $R$. Acad. Sci. Paris 315 (1992), 371-374.

[Quer 1987] J. Quer, "Corps quadratiques de 3-rang 6 et courbes elliptiques de rang 12", Comptes R. Acad. Sci. Paris 305 (1987), 215-218.
[Schoof 1983] R. Schoof, "Class groups of complex quadratic fields", Math. Comp. 43 (1983), 295-302.

[Shanks 1971] D. Shanks, "Class number, a theory of factorisation and genera", pp. 415-440 in 1969 Number Theory Institute, Proc. Sympos. Pure Math. 20, Amer. Math. Soc., Providence, RI, 1971.

[Shanks 1972] D. Shanks, "A quadratic field of prime discriminant requiring three generators for its class group, and related theory", Acta Arithm. 21 (1972), $71-87$.

[Solderitsch 1977] J. J. Solderitsch, "Quadratic fields with special class groups", Thesis, Lehigh University, 1977.

Franck Leprévost, Université Paris 7, Département de Mathématiques, Tour 45-55, 5 e étage, 2 place Jussieu, 75252 Paris Cedex 05, France (leprevot@mathp7.jussieu.fr)

Received April 7, 1993; accepted in revised form August 31 